\title{
Do Work Condition Interventions Affect Quality and Errors in Primary Care? Results from the Healthy Work Place Study
}

\author{
Mark Linzer, $M D^{1,2,3,4}$, Sara Poplau, $B A^{1,2}$, Roger Brown, $P h D^{5}$, Ellie Grossman, $M D, M P H^{6,7}$, \\ Anita Varkey, $M D^{8}$, Steven Yale, $M D^{9}$, Eric S. Williams, $P h D^{10}$, Lanis Hicks, $P h D^{11}$, Jill Wallock, $B S^{8}$, \\ Diane Kohnhorst, $\mathrm{BS}^{12}$, and Michael Barbouche, $\mathrm{MS}^{13}$
}

\begin{abstract}
${ }^{1}$ Hennepin County Medical Center, Minneapolis, MN, USA; ${ }^{2}$ Minneapolis Medical Research Foundation, Minneapolis, MN, USA; ${ }^{3}$ University of Minnesota Medical School, Minneapolis, MN, USA; 'Division of General Internal Medicine, Hennepin County Medical Center, Minneapolis, MN, USA; ${ }^{5}$ University of Wisconsin School of Medicine and Public Health and the School of Nursing, Madison, WI, USA; ${ }^{6} \mathrm{NYU}$ School of Medicine, New York, NY, USA; ${ }^{7}$ Cambridge Health Alliance, Somerville, MA, USA; ${ }^{8}$ Loyola University Medical Center and Stritch School of Medicine, Maywood, IL, USA; ${ }^{9}$ North Florida Regional Medical Center, Gainesville, FL, USA; ${ }^{10}$ Culverhouse College of Commerce, The University of Alabama, Tuscaloosa, AL, USA; ${ }^{11}$ University of Missouri, Columbia, MO, USA; ${ }^{12}$ Marshfield Clinic Research Foundation, Marshfield, WI, USA; ${ }^{13}$ Forward Health Group, Inc., Madison, WI, USA.
\end{abstract}

BACKGROUND: While primary care work conditions are associated with adverse clinician outcomes, little is known about the effect of work condition interventions on quality or safety.

DESIGN: A cluster randomized controlled trial of 34 clinics in the upper Midwest and New York City.

PARTICIPANTS: Primary care clinicians and their diabetic and hypertensive patients.

INTERVENTIONS: Quality improvement projects to improve communication between providers, workflow design, and chronic disease management. Intervention clinics received brief summaries of their clinician and patient outcome data at baseline.

MAIN MEASURES: We measured work conditions and clinician and patient outcomes both at baseline and 612 months post-intervention. Multilevel regression analyses assessed the impact of work condition changes on outcomes. Subgroup analyses assessed impact by intervention category.

KEY RESULTS: There were no significant differences in error reduction (19\% vs. $11 \%$, OR of improvement 1.84 , $95 \%$ CI $0.70,4.82, p=0.21$ ) or quality of care improvement (19 \% improved vs. $44 \%$, OR 0.62, $95 \%$ CI 0.58 , 1.21, $p=0.42$ ) between intervention and control clinics. The conceptual model linking work conditions, provider outcomes, and error reduction showed significant relationships between work conditions and provider outcomes $(p \leq 0.001)$ and a trend toward a reduced error rate in providers with lower burnout (OR 1.44, $95 \%$ CI 0.94 , 2.23, $p=0.09$ ).

LIMITATIONS: Few quality metrics, short time span, fewer clinicians recruited than anticipated.

CONCLUSIONS: Work-life interventions improving clinician satisfaction and well-being do not necessarily reduce errors or improve quality. Longer, more focused

Electronic supplementary material The online version of this article (doi:10.1007/s11606-016-3856-2) contains supplementary material, which is available to authorized users.

Received April 28, 2016

Revised July 21, 2016

Accepted August 19, 2016

Published online September 9, 2016 interventions may be needed to produce meaningful improvements in patient care.

Clinical trial registration number: ClinicalTrials.gov \# NCT02542995.

KEY WORDS: burnout; work-life; physician stress; physician burnout; work-life interventions.

$J$ Gen Intern Med 32(1):56-61

DOI: $10.1007 / \mathrm{s} 11606-016-3856-2$

(c) Society of General Internal Medicine 2016

\section{INTRODUCTION}

In the late 1990s, the Agency for Healthcare Quality and Research (AHRQ) highlighted the potential link between work conditions in doctors' offices and the quality of care they provided. ${ }^{1}$ Since then, numerous investigations have assessed these connections. ${ }^{2-4}$ The literature shows a clear relationship between work conditions and clinician outcomes such as satisfaction, stress, burnout, and intent to leave the practice, ${ }^{3,4}$ but the relationship between work conditions and patient outcomes has remained elusive.

The Minimizing Error, Maximizing Outcome (MEMO) study showed strong cross-sectional relationships between work conditions in primary care offices and clinician outcomes $^{3,5}$ but inconsistent relationships between work conditions and patient outcomes. The MEMO study revealed that lower-quality care, as measured by diabetes and hypertension control, was provided in clinics where work conditions were poorer, and that it was not necessarily the burned-out physicians who provided lower-quality care. ${ }^{3}$ These findings suggest that lower-quality care was not a function of burnout but rather of the clinic structure and culture that resulted in burnout.

The Healthy Work Place (HWP) study, launched in 2010, employed a rigorous study design to determine whether work condition interventions that led to better clinician outcomes would result in improved patient care. Previously published data from HWP on clinician outcomes showed that burnout was more frequently reduced in intervention clinics engaged 
in workflow redesign and quality improvement (QI) initiatives in chronic disease management than in control clinics (OR 5.9 and 4.8 , respectively, $p=0.02$ ). Additionally, clinics undertaking communication improvement measures or workflow redesign demonstrated more frequent improvement in professional satisfaction than control clinics (OR 3.1, $p=0.04$ ) and tended to have fewer clinicians with intentions to leave than control clinics (OR 4.2, $p=0.06$ ). ${ }^{6}$

The current analysis of HWP data asks the following questions: 1) Do clinicians in clinics undergoing work-life interventions provide higher-quality care and make fewer errors than those in control clinics? 2) Do specific types of work-life interventions, effective in improving clinician outcomes, result in higher-quality care and fewer errors? 3) Do the findings of HWP confirm the MEMO conceptual model that links work conditions to clinician reactions and patient outcomes?

\section{METHODS}

The HWP study design has been described in detail elsewhere. ${ }^{6}$ In brief, three study sites were chosen to provide a mix of urban, rural, and academic practices. Primary care clinicians $(n=165)$ were recruited from 34 clinics, and included family physicians and general internists as well as nurse practitioners and physician assistants (advanced practice providers, or APPs). Sample size calculations using intra-class correlations (ICCs) based on prior primary care work condition studies suggested that 34 clinics, the clinicians at these clinics, and 6-8 patients of each clinician would provide $80 \%$ power to answer study questions. ICCs used in these estimates ranged from 0.04 to 0.13 .

Study Design. HWP was a cluster randomized controlled trial based on the conceptual model from MEMO. ${ }^{3}$ This model directly linked clinicians' work conditions to quality of patient care. Additionally, the model showed clinicians' work conditions impacting their own reactions, which in turn influenced the quality of patient care. ${ }^{3}$ In HWP, a permuted block randomization scheme was employed. Measures of work-life and work conditions were performed at baseline and contributed to a two-page summary called the Office and Work Life (OWL) measure. OWL measures were provided to the 17 intervention clinics. The OWL included clinician perceptions of work conditions, manager data on staffing and room availability, clinician outcomes (such as satisfaction, stress, burnout, and intent to leave the practice), and patient outcomes (satisfaction, quality of care metrics, and errors). Clinic staff reviewed and discussed OWL data with research staff and chose work condition interventions specific to their OWL findings. After 6-12 months, data were collected on clinician reactions, quality of care, and errors at all 34 clinics (see consort diagram, Fig. 1.) The institutional review board at Hennepin County Medical Center and other participating sites approved the study prior to data collection. Informed consent was obtained from all enrolled participants (providers, clinic managers, and patients) by study staff.

Measures. A clinician survey was used to assess work conditions and clinician reactions. Questions were drawn from instruments used in MEMO and the Physician Worklife Study. ${ }^{7-9}$ Clinicians responded to questions about their stress, burnout, satisfaction, and intent to leave the practice within 2 years. They also provided data on work control (a 14-item scale from $\mathrm{MEMO}^{8}$ ), chaos in the clinic (a single-item measure arising from physician focus groups in $\mathrm{MEMO}^{10}$ ), time pressure during office visits ${ }^{11}$, and values alignment with leaders $^{12}$. The work control scale, described in $2002^{8}$, comprised control of workplace, medical decisions, and interruptions. The chaos measure used a single-item scale of work pace, from $1=$ calm to $5=$ hectic or chaotic, with 4 or 5 representing chaos. Values alignment included emphasis of clinic leadership on teamwork, professionalism, and revenue (each item rated as $1=$ slight/none to $4=$ great). A survey given to clinic managers provided data on clinic structure, including room availability, staffing, and care bottlenecks.

Patient data were collected through a patient survey which asked about satisfaction with medical care (using an instrument from Haas ${ }^{13}$ ). Patient data were also obtained through chart reviews by trained auditors, who collected data on diabetes and hypertension management and preventive care (lipid, alcohol, cancer, and tobacco screening). Auditors determined whether errors were made in diagnosis or in care of chronic medical conditions (e.g. not addressing continued elevation in blood pressure or hemoglobin A1C). Metrics of quality of care and error (see Table A, online supplement) were similar to those used in the MEMO study. The main measures utilized in the current analysis were clinician outcomes (satisfaction, stress, and burnout), predictive covariates (work control, chaos, and values alignment) and clinical outcomes (quality and errors).

Interventions. QI teams at intervention clinics designed interventions that addressed their OWL data. Quality improvement teams generally consisted of clinic managers and/or leaders, members of the department, and relevant providers and clinic staff (depending on the topic of the initiative). After the study was completed, team investigators used site directors' descriptions to classify QI interventions into categories. Interventions clustered predominantly in three types (see Table B, online supplement): improving communication between providers, ${ }^{2}$ workflow redesign, ${ }^{14,15}$ and targeted chronic disease management programs. ${ }^{3}$ Because many clinics addressed more than one intervention type, we were not able to assess whether specific interventions were more or less effective than others.

Analyses. The analysis considered a multi-method approach. Initially, we used a simple per-protocol regression analysis to 


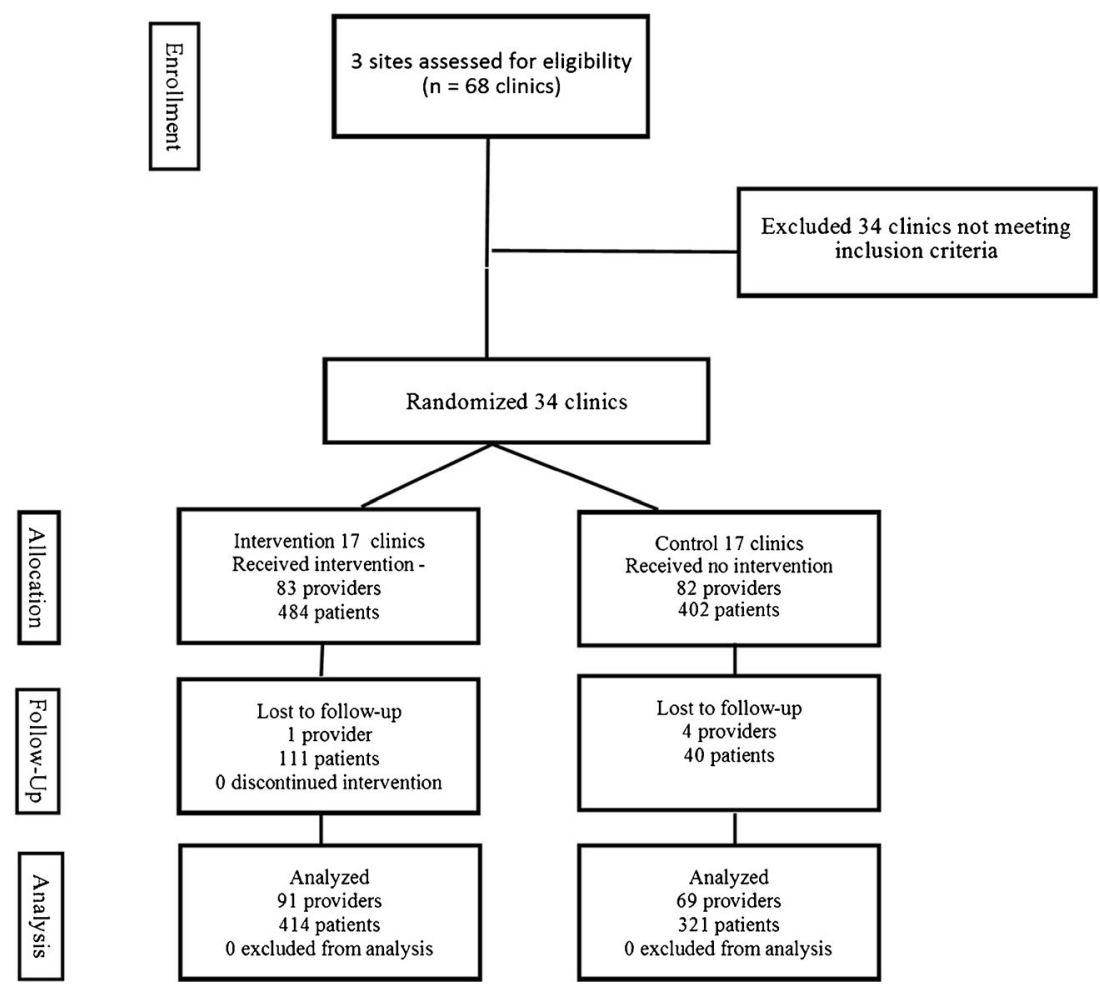

Figure 1 Consort diagram for patient flow in the HWP cluster randomized controlled trial of work-life interventions. Additional providers joined the study after allocations.

assess the effect of the interventions on the two main outcomes of improvement in errors and quality of care. We used a definition of improvement which included any positive change in error and quality counts from baseline to postintervention. Because the study was designed as a clustered randomized controlled trial with the clinic as the unit of randomization, three-level multilevel logistic regression models were used, with patients nested under health care providers, who were nested within clinics. ${ }^{16}$

Changes in specification of intervention were considered in a subgroup analysis to generate hypotheses and establish further insight into the results. ${ }^{17}$ Subgroups were specified after data collection was completed but prior to constructing the analysis. We used the three main categories of intervention types determined in the first HWP paper ${ }^{6}$ to model improvement in outcomes as a function of type of intervention. Because no intervention was designed to allow us to look at specific types of interventions, we performed this analysis to determine whether some intervention types were better than others. The first strategy involved categorizing the interventions into two subgroups of clinics: one set that used communication improvement and chronic disease management QI approaches (CI/QI) and another set that used communication improvements and workflow redesign $(\mathrm{CI} / \mathrm{W})$. The second strategy defined two clinic subgroups: one set that used communication improvements (CI) and another set that predominantly used workflow redesign (W).

Finally, we used structural equation models (SEMs) and multilevel structural probit models ${ }^{18}$ to assess the various intervention approaches and the relationships between work conditions and clinician and patient outcomes by incorporating the mediational model from MEMO. This was considered an important next step in the evolving research, as it would be the first independent validation in a randomized trial of the MEMO model linking interventions and work conditions to clinician and patient outcomes.

\section{RESULTS}

There were 165 clinicians (143 physicians and 22 APPs) and 1131 patients enrolled in the study. Of 888 patients who underwent chart audits for quality and errors at the start of the trial, 735 were available for auditing at the end of the trial (17.4\% attrition; see consort diagram, Fig. 1). Fewer patients were audited than enrolled due to withdrawal and lack of eligibility (lack of target condition, only one visit during study period, or no visits for chronic disease management). Clinicians in intervention clinics were similar to those in control sites with regard to duration of practice (14 vs. 11 years, $p=$ $0.07)$ and degree of satisfaction with their work environment (39\% vs. $52 \%$ high satisfaction, $p=0.08$ ). Clinicians at intervention clinics were more likely at baseline to want to leave their practice within 2 years (32\% vs. $17 \%, p=0.03$ ). Table 1 shows baseline provider characteristics (e.g gender, specialty, and years in practice) and patient characteristics (age, gender, race/ethnicity, insurance status, patient 
Table 1 Baseline Provider and Patient Characteristics in Healthy Work Place Trial Clinics

\begin{tabular}{|c|c|c|}
\hline Provider characteristic & $\begin{array}{l}\text { Intervention clinics } \\
(n=83)\end{array}$ & $\begin{array}{l}\text { Control clinics } \\
(n=81)\end{array}$ \\
\hline Family medicine & $33.7 \%$ & $30.0 \%$ \\
\hline Minority & $13.0 \%$ & $20.0 \%$ \\
\hline Male & $45.0 \%$ & $47.0 \%$ \\
\hline Part time & $27.0 \%$ & $21.0 \%$ \\
\hline Owner & $34.0 \%$ & $30.0 \%$ \\
\hline High satisfaction & $39.0 \%$ & $52.0 \%$ \\
\hline Intention to leave & $32.0 \%$ & $17.0 \%$ \\
\hline Years worked in practice & 14.0 & 11.4 \\
\hline Days on call & 6.2 & 5.1 \\
\hline Patient characteristic & $\begin{array}{l}\text { Intervention clinics } \\
(n=586)\end{array}$ & $\begin{array}{l}\text { Control clinics } \\
(n=545)\end{array}$ \\
\hline Mean age in years & $65.05(12.55)$ & $64.31(11.86)$ \\
\hline Male gender & $39.7 \%(233 / 586)$ & $\begin{array}{l}38.2 \%(208 / \\
544)\end{array}$ \\
\hline \multicolumn{3}{|l|}{ Race/ethnicity } \\
\hline White & $76.8 \%(448 / 583)$ & $\begin{array}{l}70.2 \%(379 / \\
540)\end{array}$ \\
\hline Black & $8.2 \%(48 / 583)$ & $\begin{array}{l}16.4 \%(89 / \\
540)\end{array}$ \\
\hline American Indian & $1.2 \%(7 / 583)$ & $0.56 \%(3 / 540)$ \\
\hline Pacific Islander & $0 \%(0 / 583)$ & $0 \%(0 / 540)$ \\
\hline Asian & $1.5 \%(9 / 583)$ & $2.5 \%(14 / 540)$ \\
\hline Other & $13.3 \%(78 / 583)$ & $\begin{array}{l}12.4 \%(67 / \\
540)\end{array}$ \\
\hline $\begin{array}{l}\text { Ethnicity } \\
\text { (non-Hispanic) }\end{array}$ & $84.2 \%(438 / 520)$ & $\begin{array}{l}86.7 \%(412 / \\
475)\end{array}$ \\
\hline $\begin{array}{l}\text { Insurance } \\
\text { status (yes) }\end{array}$ & $94.0 \%(550 / 585)$ & $\begin{array}{l}92.9 \%(504 / \\
542)\end{array}$ \\
\hline $\begin{array}{l}\text { Patient satisfaction with } \\
\text { care (scale } 1=\text { very satisfied } \\
\text { to } 5=\text { very dissatisfied })\end{array}$ & $1.38(0.52)$ & $1.38(0.55)$ \\
\hline $\begin{array}{l}\text { Patient trust in care/provider } \\
\text { (scale } 1=\text { not at all to } 5= \\
\text { completely) }\end{array}$ & $4.58(0.70)$ & $4.50(0.78)$ \\
\hline \multicolumn{3}{|l|}{ Quality measures:* } \\
\hline Overall proportion & $59.1 \%(346 / 585)$ & $\begin{array}{l}44.4 \%(242 / \\
545)\end{array}$ \\
\hline \multicolumn{3}{|l|}{ Error measures: } \\
\hline Overall proportion & $41.2 \%(241 / 585)$ & $\begin{array}{l}34.8 \%(190 / \\
545)\end{array}$ \\
\hline
\end{tabular}

* Quality and error measure scores $=\%$ with at least one quality metric achieved or error made

satisfaction, trust, and quality and error rates) in intervention versus control clinics.

Per-Protocol Analysis. Clinic-level modeling showed no statistically significant difference in error reduction between intervention and control clinics (19\% of patients improving in intervention clinics vs. $11 \%$ in control clinics, OR of improvement $1.84,95 \%$ CI $[0.70,4.82], p=0.21)$. There were also no statistically significant effects on quality outcomes (OR 0.62, $95 \%$ CI [0.58, 1.21], $p=0.42$ ).

Subgroup Analysis of Intervention Types. Intervention clinics were first defined as those concentrating on communication improvement and chronic disease QI projects (definition CI/QI, $n=10$ clinics) or clinics concentrating on communication improvement and workflow redesign initiatives (definition $\mathrm{CI} / \mathrm{W}, n=6$ clinics). Applying these definitions revealed no significant improvement in errors or quality outcomes between the intervention and control groups (e.g. $22 \%$ of patients in $\mathrm{CI} / \mathrm{QI}$ clinics showed improvement in errors vs. $11 \%$ in control clinics, $p=0.21$ ).

A second intervention definition type included clinics concentrating solely on communication improvement (definition CI, $n=9$ clinics) and clinics concentrating on workflow redesign (definition $\mathrm{W}, n=6$ clinics). Clinics using CI interventions demonstrated no statistically significant improvement in errors (OR 1.65, $95 \%$ CI $[0.58,4.69], p=0.34)$ or quality outcomes (OR 0.73, $95 \%$ CI [0.21, 2.53], $p=0.63)$. Similarly, clinics concentrating on workflow redesign interventions showed no statistically significant improvement in errors (OR 2.43, $95 \%$ CI $[0.58,10.19], p=0.22$ ) or quality outcomes (OR 0.39, $95 \% \mathrm{CI}[0.07,2.26], p=0.30)$.

Structural Probit Analysis. The probit model assessment found support for the MEMO model, with work control influencing reductions in provider stress $(p<0.001)$ and improvements in provider satisfaction $(p<0.001)$. In turn, provider stress was associated with higher burnout $(p<0.001)$, and provider satisfaction led to reduced burnout $(p=0.001)$. Figure 2 (see online supplement) demonstrates the relationships between interventions, work conditions (work control), clinician outcomes (stress, satisfaction, and burnout), and error improvement. This model found a borderline statistically significant effect on error improvement with reductions in burnout (OR 1.44, $95 \%$ CI $[0.94,2.23], p=0.09)$. Of note, there was a decrease in work control due to the interventions (approximately 0.2 standard deviations), which may have attenuated their impact.

\section{CONCLUSIONS}

The HWP study demonstrates that interventions in work-life can improve clinician reactions such as burnout and professional satisfaction, ${ }^{6}$ but that medical errors and quality of care are more resistant to change. The specific interventions in HWP focused on workflow redesign, ${ }^{14,19}$ improvement in communication between provider groups, ${ }^{2,20,21}$ and chronic disease management QI programs. ${ }^{22}$ The study did not identify significant differences in medical errors or quality outcomes, regardless of the intervention adopted. Several explanations could account for the findings from this study. First, programs to improve quality may lag behind clinician worklife while clinician concerns such as burnout are being addressed. Once burnout is reduced, clinics may then more effectively focus on initiatives related to quality outcomes. Longer and more focused interventions may be required to reduce medical errors and improve quality of care. Second, clinicians may have been less focused on medical errors or quality outcomes due to other mechanisms for addressing these measures within the intervention and control clinics.

Patient safety and medical errors in ambulatory clinic settings are areas of national and international concern. ${ }^{23}$ Since 
the seminal report "To Err is Human" by the Institute of Medicine ${ }^{24}$ most error reduction programs have targeted inpatient settings. However, it is likely that the substantially broader scope of outpatient medicine would allow for far more errors and thus a greater impact if structural determinants of and activities for preventing outpatient mistakes could be discerned. The types of errors studied in HWP (predominantly lack of medication adjustment when blood pressure or diabetes was out of control and failure to perform designated cancer screening activities) are prevalent and difficult to correct. Popup reminders have been built to reduce them, but clinicians suffer from "reminder fatigue," and primary care visits afford little time to attend to such issues. ${ }^{25}$

The structural models confirmed the conceptual model from MEMO linking work-life changes to significant improvements in clinician outcomes, but identified only a trend toward greater medical error reduction associated with the interventions. This model originated in 2001 as part of the Physician Worklife Study, where cross-sectional ties were demonstrated between background characteristics such as work hours and practice type with work conditions (control, work-home interference) and stress, satisfaction, and burnout. ${ }^{26}$ The model was validated in groups of both Dutch and US physicians, and explained $50 \%$ of the variance in burnout in data sets from both countries. ${ }^{26}$ The model evolved in 2009 during $\mathrm{MEMO}^{3}$ to incorporate additional work conditions (such as time pressure, chaos, and organizational culture) and patient outcomes (satisfaction, quality, and safety). The analyses in HWP confirm in a randomized study design the basic structure of the model linking work conditions to stress and burnout, while adding the impact of interventions on clinician well-being and a possible indirect connection to patient outcomes. The linear model structure in Figure 2 can be of benefit for investigators who wish to alter work conditions as a means of improving additional patient outcomes such as quality of care or patient satisfaction.

HWP was limited by brevity of the intervention period (approximately 12 months), which was a short timeline for interventions to produce observable changes in quality. Intervention types were varied, and it was uncertain how well the interventions as implemented adhered to the original plans. Formal patient response rates were unavailable, although the percentage of patients enrolled who completed their surveys exceeded $90 \%$ at all three sites. The three-level hierarchical modeling structure, while appropriate for a randomized trial, allows for inflation in standard errors, thus reducing the likelihood of showing statistically significant differences. In addition, there were fewer clinicians recruited than anticipated (69\% of the clinicians needed for $80 \%$ power), leading to a lack of sensitivity for detecting significant differences between groups. Finally, it is possible that other interventions adopted in control clinics diminished the chances for a significant difference in impact between the two groups.

In summary, HWP has shown that work-life changes can lead to meaningful change in clinician outcomes, but that these changes do not necessarily translate into improved patient care. Improvements in quality and patient safety may require more focused and sustainable programs. Interventions will potentially be more effective in clinicians with lower burnout who are better equipped for sustained engagement in QI activities.

Data Accuracy: Drs. Linzer and Brown had full access to all the data in the study and take responsibility for the integrity of the data and the accuracy of the data analysis.

Author Contributions: Drs. Linzer, Brown, Grossman, Varkey, Williams, and Hicks and Mr. Barbouche contributed to the study conception or design. Drs. Linzer, Brown, Grossman, Varkey and Yale, Ms. Wallock, and Ms. Kohnhorst contributed to data acquisition, analysis, or interpretation. Drs. Brown and Linzer, Ms. Poplau, and Ms. Kohnhorst drafted the manuscript. Drs. Brown, Grossman, Varkey, Yale, Williams, and Hicks and Ms. Poplau, Ms. Wallock, Ms. Kohnhorst, and Mr. Barbouche critically revised the manuscript for important content. Dr. Brown performed the statistical analyses. Drs. Linzer, Grossman, and Varkey and Ms. Poplau obtained funding. Administrative, technical, or material support was provided by Drs. Yale, Williams (quality improvement work), and Hicks (economics) and by Ms. Kohnhorst and Mr. Barbouche (learning community and quality measurement). Drs. Grossman, Varkey, and Yale provided supervision. Ms. Williams and Ms. Kohnhorst provided additional support through conference calls and ongoing project review.

Corresponding Author: Mark Linzer, MD; Division of General Internal MedicineHennepin County Medical Center, 701 Park Avenue (P7), Minneapolis, MN, USA (e-mail: mark.linzer@hcmed.org).

\section{Compliance with Ethical Standards:}

Funders: This research was supported by the Agency for Healthcare Research and Quality (AHRQ) grant no. 5R18-HS018160-03. The funder had no role in the design, conduct, analysis, or publication of the study.

Conflict of Interest: Dr. Hicks acknowledges royalties from a textbook. Mr. Barbouche is the CEO of Forward Health Group, Inc., owns stock in the company, and has patents pending. The other authors declare that they have no conflict of interest.

\section{REFERENCES}

1. Eisenberg JM, Bowman CC, Foster NE. Does a healthy health care workplace produce higher-quality care? Jt Comm J Qual Patient Saf. 2001;27(9):444-57.

2. Dunn PM, Arnetz BB, Christensen JF, Homer L. Meeting the imperative to improve physician well-being: assessment of an innovative program. J Gen Intern Med. 2007;22(11):1544-52. doi:10.1007/s11606-007-0363-5.

3. Linzer M, Manwell LB, Williams ES, et al. Working conditions in primary care: physician reactions and care quality. Ann Intern Med. 2009; 151(1):28-36. W6-W9.

4. Reid RJ, Coleman K, Johnson EA, et al. The group health medical home at year two: cost savings, higher patient satisfaction, and less burnout for providers. Health Aff Proj Hope. 2010;29(5):835-43. doi:10.1377/hlthaff. 2010.0158

5. Manwell LB, Williams ES, Babbott S, Rabatin JS, Linzer M. Physician perspectives on quality and error in the outpatient setting. WMJ Off Publ State Med Soc Wis. 2009;108(3): 139-44.

6. Linzer M, Poplau S, Grossman E, et al. A cluster randomized trial of interventions to improve work conditions and clinician burnout in primary care: results from the Healthy Work Place (HWP) study. J Gen Intern Med. 2015;30(8):1105-11. doi:10.1007/s11606-015-3235-4.

7. Williams ES, Konrad TR, Linzer M, et al. Refining the measurement of physician job satisfaction: results from the physician worklife survey. Med Care. 1999;37(11):1140-54.

8. Linzer M, Gerrity M, Douglas JA, McMurray JE, Williams ES, Konrad TR. Physician stress: results from the physician worklife study. Stress Health. 2002;18(1):37-42. doi:10.1002/smi.917. 
9. Konrad TR, Williams ES, Linzer M, et al. Measuring physician job satisfaction in a changing workplace and a challenging environment. Med Care. 1999;37(11):1174-82.

10. Perez HR, Beyrouti M, Bennett $\mathbf{K}$, et al. Chaos in the clinic: characteristics and consequences of practices perceived as chaotic. $J$ Healthc Qual. 2015.

11. Linzer M, Konrad TR, Douglas J, et al. Managed care, time pressure, and physician job satisfaction: results from the physician worklife study. J Gen Intern Med. 2000; 15(7):441-50.

12. Kralewski JE, Wingert TD, Barbouche MH. Assessing the culture of medical group practices. Med Care. 1996;34(5):377-88.

13. Haas JS, Cook EF, Puopolo AL, Burstin HR, Cleary PD, Brennan TA. Is the professional satisfaction of general internists associated with patient satisfaction? J Gen Intern Med. 2000;15(2):122-8.

14. Bodenheimer TS, Smith MD. Primary care: proposed solutions to the physician shortage without training more physicians. Health Aff Proj Hope. 2013;32(11):1881-6. doi:10.1377/hlthaff.2013.0234.

15. Bodenheimer T, Sinsky C. From triple to quadruple aim: care of the patient requires care of the provider. Ann Fam Med. 2014;12(6):573-6. doi:10.1370/afm.1713.

16. Goldstein $\mathbf{H}$. Nonlinear multilevel models, with an application to discrete response data. Biometrika. 1991;78(1):45-51. doi:10.2307/2336894.

17. Adams KF Jr. Post hoc subgroup analysis and the truth of a clinical trial. Am Heart J. 1998;136(5):753-8. doi:10.1016/S0002-8703(98)70116-4.

18. Muthén B. A structural Probit model with latent variables. J Am Stat Assoc. 1979;74(368):807-11. doi:10.1080/01621459.1979.10481034.
19. Sinsky CA, Beasley JW, Simmons GE, Baron RJ. Electronic health records: design, implementation, and policy for higher-value primary care. Ann Intern Med. 2014;160(10):727-8. doi:10.7326/M13-2589.

20. Gittell JH, Weinberg D, Pfefferle S, Bishop C. Impact of relational coordination on job satisfaction and quality outcomes: a study of nursing homes. Hum Resour Manag J. 2008;18(2):154-70. doi:10.1111/j.17488583.2007.00063.x.

21. Gittell JH. Coordinating mechanisms in care provider groups: relational coordination as a mediator and input uncertainty as a moderator of performance effects. Manag Sci. 2002;48(11):1408-26.

22. West CP, Dyrbye LN, Rabatin JT, et al. Intervention to promote physician well-being, job satisfaction, and professionalism: a randomized clinical trial. JAMA Intern Med. 2014;174(4):527-33. doi:10.1001/jamainternmed. 2013.14387.

23. Scheepers RA, Boerebach BCM, Arah OA, Heineman MJ, Lombarts KMJMH. A systematic review of the impact of physicians' occupational well-being on the quality of patient care. Int J Behav Med. March 2015:116. doi: 10.1007/s12529-015-9473-3.

24. Institute of Medicine. To err is human: Building a safer health system, 1999. http://www.nap.edu/catalog/9728/to-err-is-human-building-asafer-health-system. Accessed 20 May 2015.

25. Linzer M, Bitton A, Tu S-P, et al. The end of the 15-20 minute primary care visit. J Gen Intern Med. 2015. doi:10.1007/s11606-015-3341-3.

26. Linzer M, Visser MR, Oort FJ, et al. Predicting and preventing physician burnout: results from the United States and the Netherlands. Am J Med. 2001;111(2):170-5 